\title{
Site-Specific TEM Specimen Preparation of Samples with Sub-Surface Features
}

\author{
Julia I. Deitz, ${ }^{1}$ Santino D. Carnevale, ${ }^{2}$ David W. McComb, ${ }^{1,3}$, Steven A. Ringel, ${ }^{2,3}$, Tyler J. Grassman ${ }^{1,2}$ \\ ${ }^{1}$ Dept. of Materials Science \& Engineering, The Ohio State University, Columbus, OH, 43210, USA \\ ${ }^{2}$ Dept. of Electrical \& Computer Engineering, The Ohio State University, Columbus, OH, 43210, USA \\ ${ }^{3}$ Institute for Materials Research, The Ohio State University, Columbus, OH, 43210, USA
}

Characterization of crystalline defects has traditionally been performed in the TEM, as it has the required capabilities in terms of sensitivity and resolution to ensure sufficient accuracy. However, TEM analysis requires electron transparency, which requires preparation of thin specimens, typically achieved using focus ion beam (FIB)-based foil extraction and thinning. For specimens with features that cannot be seen on the surface, samples extracted via FIB are effectively selected "blindly." That is, the area from where the sample is extracted is, for the most part, randomly selected, and there is little or no prior knowledge as to what features will be included in the small, and extremely thin, region (approximately $10 \mu \mathrm{m} \times 5 \mu \mathrm{m} \times 100 \mathrm{~nm}$ ). Such a small sample can provide a poor statistical representation of the material as a whole, and it does not allow for a desired feature to be obtained in an efficient manner. Here, we develop a method to locate sub-surface features using electron channeling contrast imaging (ECCI) in order to extract site-specific TEM specimens using the dual beam focused ion beam (FIB) instrument.

ECCI, an alternative method for characterization to TEM, has begun to gain traction as a technique for the visualization of subsurface defects in crystalline materials, due in part to work at OSU on its application to epitaxial III-V compound semiconductor materials [1][2]. This powerful technique is performed using a field-emission scanning electron microscope (SEM) equipped with either a backscatter or forescatter electron (BSE, FSE) detector. Recently, we have demonstrated ECCI performed using the secondary electron (SE) detector as well. The ability to detect and image subsurface features with a standard SE detector means that is it now possible to alleviate the problem of unguided sample extraction with the FIB. It is standard for any FIB to have an SE detector, but not necessarily a BSE detector, meaning that ECCI can be performed on a sample in the FIB, and defects and features can be detected prior to extraction. Once a desired area is found, it can be marked with platinum and subsequently prepared for TEM characterization. This proposed method effectively eliminates the risk of "guess and check" sample preparation. Instead of merely hoping that the subject of interest will happen to have been captured via the "blind" FIB approach, where a TEM foil is taken from within the sample without any real guidance, we can pick the exact area to extract based upon the fact that we already know the subject of interest is actually there.

This contribution serves to develop this ECCI-FIB technique for the extraction of site-specific TEM specimens with a FIB. All ECCI in this work was performed on as-grown samples, using either an FEI Helios or Nova NanoLab dual-beam instrument fitted with a secondary electron (SE) detector, using an accelerating voltage of $30 \mathrm{kV}$, a spot size of $5(2.4 \mathrm{nA})$, and a working distance of $15 \mathrm{~mm}$.

ECCI-FIB is first demonstrated on a GaP (50 nm layer) on Si sample occupied with a high density of misfit dislocations. Figure 1 shows three separate images taken with the SE detector in the FIB over the same area. Figure 1(a) is taken at standard surface-normal, surface-sensitive non-diffractive SE geometry, where sub-surface features are not expected to be seen. Figure 1(b) has a diffraction vector of $\mathbf{g}=\overline{2} 20$, while 1 (c) has the inverse diffraction condition of $\mathbf{g}=2 \overline{2} 0$. It follows that 1 (c) misfit 
dislocations should show inverse contrast to 1(b), and indeed this is confirmed, showing the SE diffraction follows the same mechanisms as BSE and TEM diffraction. Misfits that appear black in 1(b) appear white in 1(c) and vise versa. We will also present SE FIB work for more complex systems and different types of defects.

When used in practice, after locating a feature of defect of interest with ECCI, a small amount of platinum can be deposited - to mark the location - with the e-beam, followed by standard ion beam platinum deposition. Subsequently, site specific FIB extraction can occur. In this presentation, we will discuss further details of this method to ensure accurate extraction with minimized beam damage.

Conclusion: We demonstrate the use of ECCI for site specific extraction in the FIB for sub surface features. The project presented here serves to develop this method, ECCI-FIB, into an extensible tool for use in a wide range of research projects where sub-surface features need to be captured within the prepared specimens for TEM analysis. This application of ECCI removes risk of blind FIB work, giving guidance to the FIB user for accelerated research.

\section{References:}

[1] S. Carnevale, J. Deitz, T. Grassman, J. Carlin, Y. Picard, M. De Graef, S. Ringel, "Rapid misfit dislocation characterization in heteroepitaxial III-V/Si thin films by electron channeling contrast imaging," Applied Physics Letters 104(23), 232111 (2014).

[2] S. Carnevale, J. Deitz, T. Grassman, J. Carlin, Y. Picard, M. De Graef, S. Ringel, "Applications of Electron Channeling Contrast Imaging for the Rapid Characterization of Extended Defects in III-V/Si Heterostructures," IEEE Journal of Photovoltaics, accepted (2014); Early Access: http://dx.doi.org/10.1109/JPHOTOV.2014.2379111.

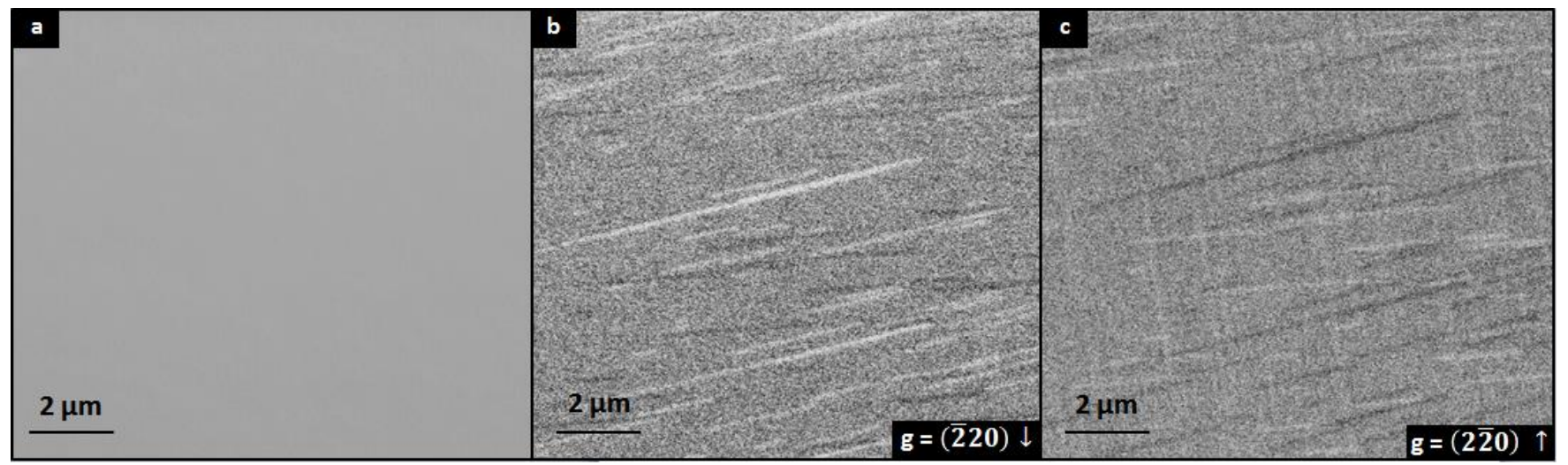

Figure 1: SE images of GaP/Si sample in the FIB, over the same area. 1(a), the SE is at standard, nondiffracting geometry, while (b) has a diffraction condition of $g=(\overline{2} 20)$ and (c) with $g=(2 \overline{2} 0)$. 